\title{
Classical and quantum two-dimensional anisotropic Heisenberg antiferromagnets
}

\author{
M. Holtschneider, ${ }^{1}$ S. Wessel, ${ }^{2}$ and W. Selke ${ }^{1}$ \\ ${ }^{1}$ Institut für Theoretische Physik, RWTH Aachen, 52056 Aachen, Germany \\ ${ }^{2}$ Institut für Theoretische Physik III, Universität Stuttgart, 70550 Stuttgart, Germany
}

\begin{abstract}
The classical and the quantum, spin $S=\frac{1}{2}$, versions of the uniaxially anisotropic Heisenberg antiferromagnet on a square lattice in a field parallel to the easy axis are studied using Monte Carlo techniques. For the classical version, attention is drawn to biconical structures and fluctuations at low temperatures in the transition region between the antiferromagnetic and spin-flop phases. For the quantum version, the previously proposed scenario of a first-order transition between the antiferromagnetic and spin-flop phases with a critical endpoint and a tricritical point is scrutinized.
\end{abstract}

PACS numbers: 75.10.Hk, 75.10.Jm, 75.40.Mg, 05.10.Ln

\section{INTRODUCTION}

Uniaxially anisotropic Heisenberg antiferromagnets in an external field along the easy axis have attracted much interest, both theoretically and experimentally, due to their interesting structural and critical properties. In particular, they display a spin-flop phase, and multicritical behavior occurs at the triple point of the antiferromagnetic (AF), spin-flop (SF) and paramagnetic phases $1,2,3,4,5,6,7,8,9,10$

A prototypical model for such antiferromagnets is the XXZ model, with the Hamiltonian

$$
\mathcal{H}=J \sum_{(i, j)}\left[\Delta\left(S_{i}^{x} S_{j}^{x}+S_{i}^{y} S_{j}^{y}\right)+S_{i}^{z} S_{j}^{z}\right]-H \sum_{i} S_{i}^{z}
$$

where the sum runs over neighboring spins of a cubic, dimension $d=3$, or square lattice, $d=2$. The coupling constant $J$ and the field $H$ are positive; the anisotropy parameter $\Delta$ may range from zero to one. Furthermore, $S_{i}^{x}, S_{i}^{y}$, and $S_{i}^{z}$ denote the spin components at lattice site $i$.

For the three-dimensional case, early renormalization group arguments $\frac{1}{\underline{\underline{m}}}$ and Monte Carlo simulations ${ }^{3}$ suggested that the triple point is a bicritical point with $O(3)$ symmetry. Only a few years ago, this scenario has been questioned, based on high-order perturbative renormalization group calculations. $\stackrel{11}{\underline{1}}$ It has been predicted that there may be either a first order transition, or that the 'tetracritical biconical' $\stackrel{1}{=}$ fixed point, due to an intervening 'mixed' or 'biconical' phase in between the $\mathrm{AF}$ and SF phases $12,13,14$, may be stable.

In two dimensions, conflicting predictions on the nature of the triple point have been put forward recently $15,16,17,18$, when analyzing the classical version of the above model with spin vectors of unit length, and the quantum version with spin $S=\frac{1}{2}$.

Indeed, in the classical case, simulational evidence for a narrow (disordered) phase between the AF and SF phases has been presented ${ }^{15}$, extending presumably down to zero temperature $\frac{16}{6}$ On the other hand, in the quantum case, based on simulations as well, a direct transition of first order between the $\mathrm{AF}$ and $\mathrm{SF}$ phases has been argued to occur at low temperatures $\underline{\underline{18,19,20}}$
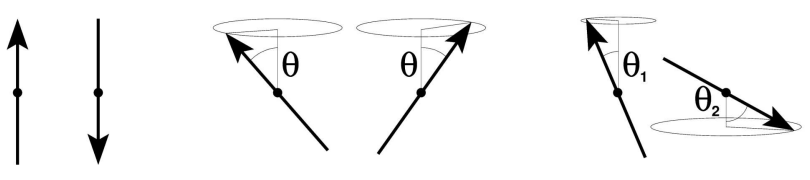

FIG. 1: Ground state configurations of the classical model sketched by the directions of spins on the two sublattices (i.e. at neighboring sites), from left to right: AF, SF, and biconical state. The circles denote the trivial degeneracy in the $x y$ plane.

Obviously, experimental data have to be viewed with care because deviations from the XXZ Hamiltonian, Eq. (11), such as crystal field anisotropies or longer-range interactions, may affect relevantly the critical behavior of the triple point,$\frac{5,12,13,14,21,22}{}$

In the following, we present results from large-scale Monte Carlo simulations of the XXZ model on a square lattice for both the classical and the quantum variant. In the quantum Monte Carlo simulations, the method of the stochastic series expansion (SSE) ${ }^{23}$ is used, and the standard Metropolis algorithm is applied for the classical case. The simulations are augmented by a ground-state analysis of the classical model, showing the significance of biconical structures. The outline of the paper is as follows: First we shall discuss our findings on the classical model, followed by a section on the quantum version of the XXZ model. A summary concludes the paper.

\section{CLASSICAL MODEL}

The ground states of the classical model on a square lattice, see Hamiltonian (1), can be determined exactly. The AF structure is stable for magnetic fields below the critical value

$$
H_{\mathrm{c} 1}=4 J \sqrt{1-\Delta^{2}}
$$

while for larger fields the SF state is energetically favorable. At $H_{\mathrm{c} 1}$, the tilt angle $\theta_{\mathrm{SF}}$ of the SF structures, see 


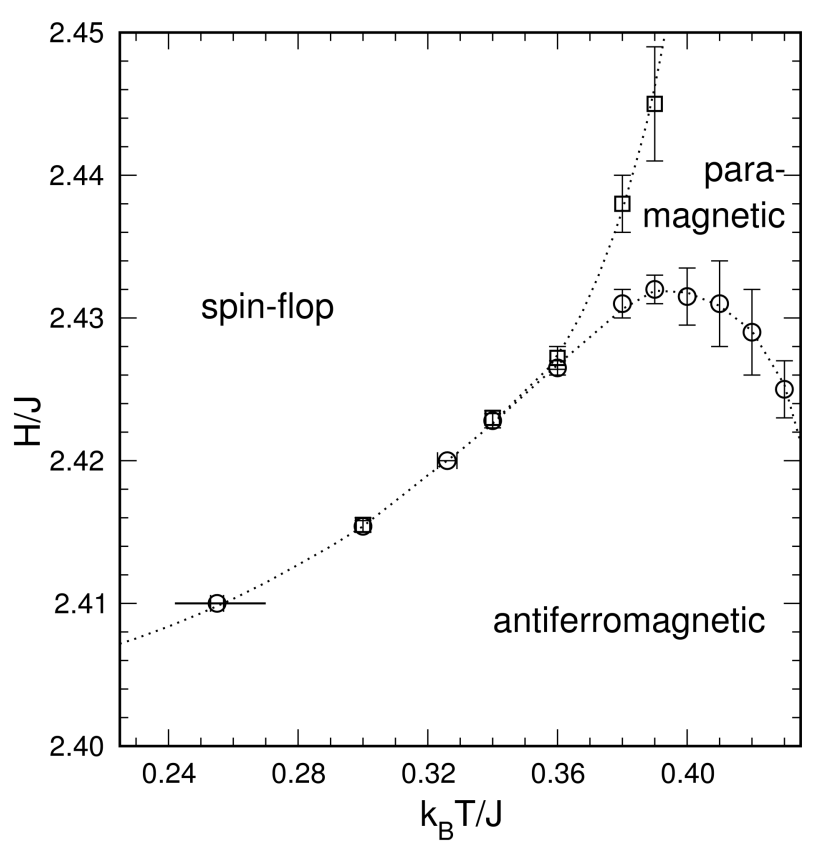

FIG. 2: Detail of the phase diagram of the XXZ model on a square lattice with $\Delta=\frac{4}{5}$, see Ref. 15. Squares refer to the boundary of the SF, circles to that of the AF phase. The solid line refers to the magnetic field $H / J=2.41$, where the probability distribution $P\left(\theta_{m}, \theta_{n}\right)$, depicted in Fig. 3. has been obtained. Here and in the following figures error bars are shown only if the errors are larger than the symbol size and dotted lines are guides to the eye.

Fig. [1] is given by

$$
\theta_{\mathrm{SF}}=\arccos \sqrt{\frac{1-\Delta}{1+\Delta}}
$$

Increasing the field beyond $H_{\mathrm{c} 2}=4 J(1+\Delta)$, all spins perfectly align in the $z$-direction.

At the critical field $H_{\mathrm{c} 1}$, see Eq. (2), the ground state is degenerate in the $\mathrm{AF}$, the $\mathrm{SF}$, and biconical structures, as illustrated in Fig. 1. This degeneracy in the biconical configurations, following from straightforward energy considerations, seems to have been overlooked in the previous analyses. The structures may be described by the tilt angles, $\theta_{1}$ and $\theta_{2}$, formed between the directions of the spins on the two sublattices of the antiferromagnet and the easy axis. For a given value of $\theta_{1}$, the other angle $\theta_{2}$ is fixed by

$$
\theta_{2}=\arccos \left(\frac{\sqrt{1-\Delta^{2}}-\cos \theta_{1}}{1-\sqrt{1-\Delta^{2}} \cos \theta_{1}}\right) .
$$

Obviously, the biconical configurations transform the AF into the SF state: The spins on the "up-sublattice" of the $\mathrm{AF}$ structure, with the spins pointing into the direction of the field, may be thought of to vary from $\theta_{1}=0$ to $\pm \theta_{\mathrm{SF}}$, while the spins on the "down-sublattice" vary simultaneously from $\theta_{2}=\pi$ to $\mp \theta_{\mathrm{SF}}$. Accordingly, $\theta_{1}$ determines

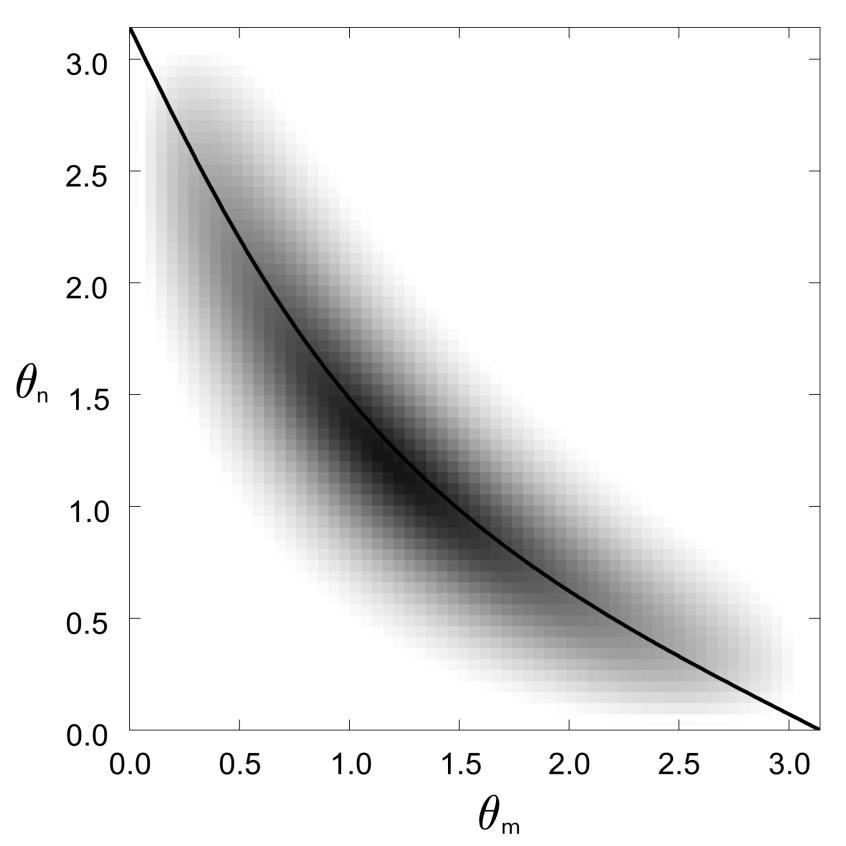

FIG. 3: Joint probability distribution $P\left(\theta_{m}, \theta_{n}\right)$ showing the correlations between the tilt angles $\theta_{m}$ and $\theta_{n}$ on neighboring sites $m$ and $n$ for a system of size $L=80$ at $H / J=2.41$, $k_{B} T / J=0.255$, and $\Delta=\frac{4}{5} . P\left(\theta_{m}, \theta_{n}\right)$ is proportional to the gray scale. The superimposed black line depicts the relation between the two angles in the biconical ground state, see Eq. (41).

uniquely $\theta_{2}$ and vice versa. Apart from this continuous degeneracy in $\theta_{1}$ (or $\theta_{2}$ ), there is an additional rotational degeneracy of the biconical configurations in the spin components perpendicular to the easy axis, the $x y$ components, as for the SF structure, see Fig. (11). These components are, of course, antiferromagnetically aligned at neighboring sites.

To study the possible thermal relevance of the biconical structures at $T>0$, we performed Monte Carlo simulations analyzing the joint probability distribution $P\left(\theta_{m}, \theta_{n}\right)$ for having tilt angles $\theta_{m}$ and $\theta_{n}$ at neighboring sites, $m$ and $n$. For comparison with the previous studies $\frac{3,15,16}{16}$ we set $\Delta=\frac{4}{5}$, leading to the phase diagram depicted in Fig. 2. For example, fixing the field at $H=2.41 J$, we observed at $k_{B} T / J \approx 0.255$ an Ising-type transition on approach from higher temperatures and a Kosterlitz-Thouless-type transition on approach from the low-temperature side, extending our corresponding previous findings $\frac{15}{}$ to even lower temperatures, and in agreement with recent results. $\stackrel{16}{\underline{1}}$ Indeed, as depicted in Fig. 3. in that part of the phase diagram, being in the vicinity of the very narrow intervening, supposedly disordered phase, the joint probability $P\left(\theta_{m}, \theta_{n}\right)$ exhibits a line of local maxima following closely Eq. (44), obtained for the ground state. That behavior is largely independent of the size of the lattices we studied. Similar signatures of the biconical structures are observed in 


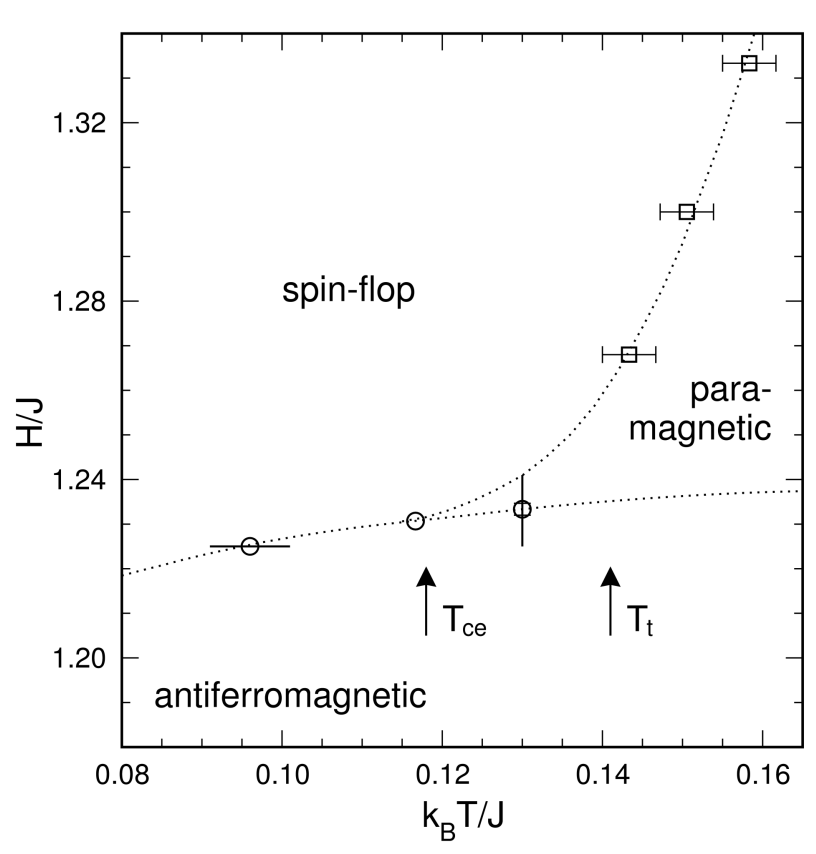

FIG. 4: Phase diagram of the XXZ Heisenberg antiferromagnet with spin- $\frac{1}{2}$ and $\Delta=\frac{2}{3}$. The straight solid lines denote the choices of parameters where our very extensive simulations, discussed in the text, have been performed. The arrows mark the previously ${ }^{18}$ suggested locations of the tricritical point $\left(T_{\mathrm{t}}\right)$ and the critical endpoint $\left(T_{\mathrm{ce}}\right)$.

the simulations at nearby temperatures, when fixing the field at $H=2.41 J$, as well as in the vicinity of the entire transition region between the $\mathrm{AF}$ and $\mathrm{SF}$ phases, see Fig. 2] at higher fields and temperatures.

Accordingly, we tend to conclude that biconical fluctuations are dominating in the narrow intervening phase. Whether that phase exists as a disordered phase down to the ground state or whether there is a stable biconical phase in two dimensions, remain open questions, being beyond the scope of this article.

Note that our additional Monte Carlo simulations for the anisotropic XY antiferromagnet in a field on a square lattice show that the analogues of 'biconical' structures (the orientation of the spins being now given by the two tilt angles only) and fluctuations play an important role near the transition regime between the AF and SF phases in that case as well. In fact, Eq. (4) provides an excellent guidance for interpreting our simulational data similar to the ones presented in Fig. 3.

\section{QUANTUM XXZ MODEL}

The aim of the study on the quantum version, $S=\frac{1}{2}$, of the XXZ model, Eq. (1), has been to check the previously suggested scenario of a first-order phase transition between the AF and SF phases extending up to a critical endpoint and with a tricritical point on the AF phase boundary, see Fig. 4 .

We performed quantum Monte Carlo (QMC) simulations in the framework of the stochastic series expansion (SSE) ${ }^{23}$ using directed loop updates $\stackrel{24}{ }$. We consider square lattices of $L \times L$ sites with the linear dimension $L$ ranging from 2 to 150 , employing full periodic boundary conditions. Defining, as usual, $\stackrel{23}{a}$ a single QMC step as one diagonal update followed by the construction of several operator-loops, each individual run typically consists of $10^{6}$ steps and is preceded by at least $2 \cdot 10^{5}$ steps for thermal equilibration. Averages and error bars are obtained by taking into account results of several, ranging from 8 to 32 , Monte Carlo runs, choosing different initial configurations and random numbers. Especially for large systems and low temperatures we additionally utilize the technique of parallel tempering (or exchange Monte Carlo) $)^{25,26}$ to enable the simulated systems to overcome the large energy barriers between configurations related to different phases more frequently. We typically work with a chain of 16 to 32 configurations in parallel which are simulated at different equally spaced temperature or magnetic field values allowing for an exchange of neighboring configurations after a constant number of QMC steps. The achieved reduction of the autocorrelation times, e.g. of the different magnetizations discussed below, amounts up to several orders of magnitude and therefore results in significantly smaller correlations between subsequent measurements which, in turn, allows for shorter simulation times.

To determine the phase diagram and to check against previous work ${ }^{18}$, we calculated various physical quantities including the $z$-component of the total magnetization,

$$
M^{z}=\frac{1}{L^{2}} \sum_{i}\left\langle S_{i}^{z}\right\rangle
$$

and the square of the $z$-component of the staggered magnetization,

$$
\left(M_{\mathrm{st}}^{z}\right)^{2}=\frac{1}{L^{2}}\left[\sum_{i_{a}}\left\langle S_{i_{a}}^{z}\right\rangle-\sum_{i_{b}}\left\langle S_{i_{b}}^{z}\right\rangle\right]^{2},
$$

summing over all sites, $i_{a}$ and $i_{b}$, of the two sublattices of the antiferromagnet. A useful quantity in studying the SF phase is the spin-stiffness $\rho_{s}$ which is related to the change of the free-energy on imposing an infinitesimal twist on all bonds in one direction of the lattice. In QMC simulations the spin-stiffness can conveniently be measured by the fluctuations of the winding numbers $W_{x}$ and $W_{y} \stackrel{23}{\stackrel{2}{ }}$

$$
\rho_{s}=\frac{k_{B} T}{2}\left(W_{x}^{2}+W_{y}^{2}\right) .
$$

The winding numbers themselves are given by

$$
W_{\alpha}=\frac{1}{L}\left(N_{\alpha}^{+}-N_{\alpha}^{-}\right),
$$




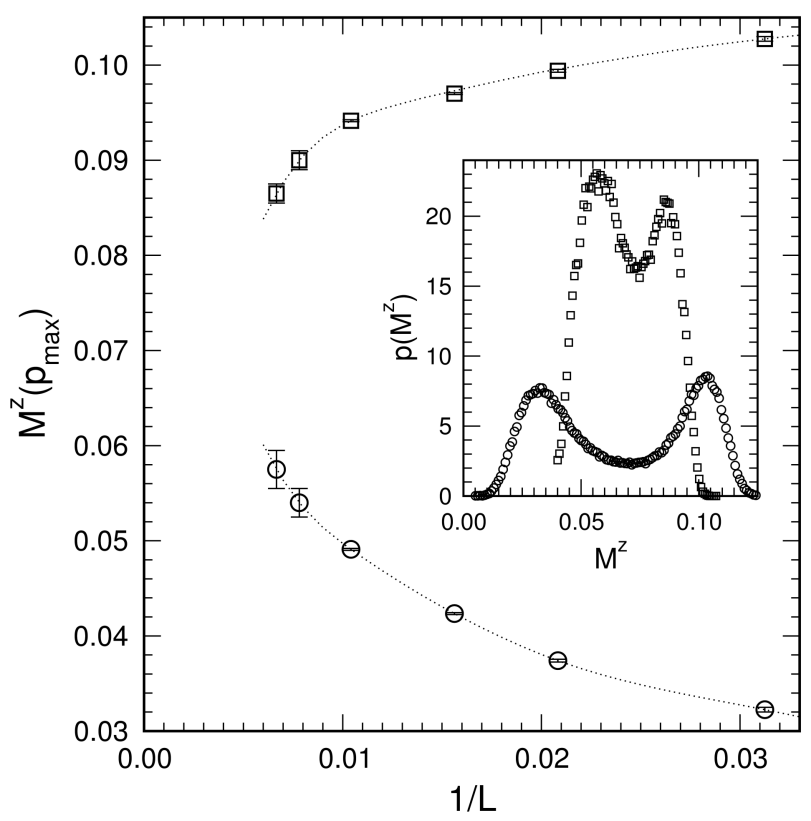

FIG. 5: Positions of the maxima of the magnetization histograms as a function of the inverse system size. The inset exemplifies two histograms for systems of size $L=32$ (circles) and $L=150$ (squares) at $k_{B} T / J=0.13$ and the coexistence fields $H / J=1.23075$ and $H / J=1.232245$.

where $N_{\alpha}^{+}$and $N_{\alpha}^{-}$denote the number of operators $S_{i}^{+} S_{j}^{-}$and $S_{i}^{-} S_{j}^{+}$in the SSE operator sequence with a bond $\langle i, j\rangle$ in the $\alpha$-direction, $\alpha \in\{x, y\}$.

All data for the quantum model presented here are obtained at an anisotropy parameter of $\Delta=\frac{2}{3}$ to allow for comparison with previous findings 15,18 . The phase diagram in the region of interest, where all three phases, the $\mathrm{AF}$, the $\mathrm{SF}$, and the paramagnetic phase occur, is displayed in Fig. 4 .

The earlier study 18 asserted a phase diagram with a tricritical point at $k_{B} T_{\mathrm{t}} / J \approx 0.141$ and a direct first-order transition between the $\mathrm{SF}$ and $\mathrm{AF}$ phases below the criti-

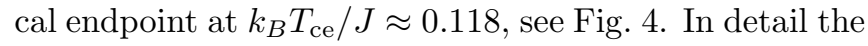
authors identified a first-order AF to paramagnetic transition at $k_{B} T / J=0.13$ by means of an analysis of the magnetization histograms $p\left(M^{z}\right)$. We studied that case, improving the statistics and considering even larger lattice sizes. Indeed, as expected for a discontinuous change of the magnetization, the histograms of finite systems are confirmed to display two distinct maxima corresponding to the ordered and the disordered phase in the vicinity of the AF phase boundary (see inset of Fig. 5). Note however, that such a two-peak structure can also be found for small systems at a continuous transition, with a single peak in the thermodynamic limit. Thence, a careful finite-size analysis is needed to, possibly, discriminate the two different scenarios. We simulated lattice sizes with up to $150 \times 150$ spins adjusting the magnetic field such that coexistence of the phases, i.e. equal weight of the

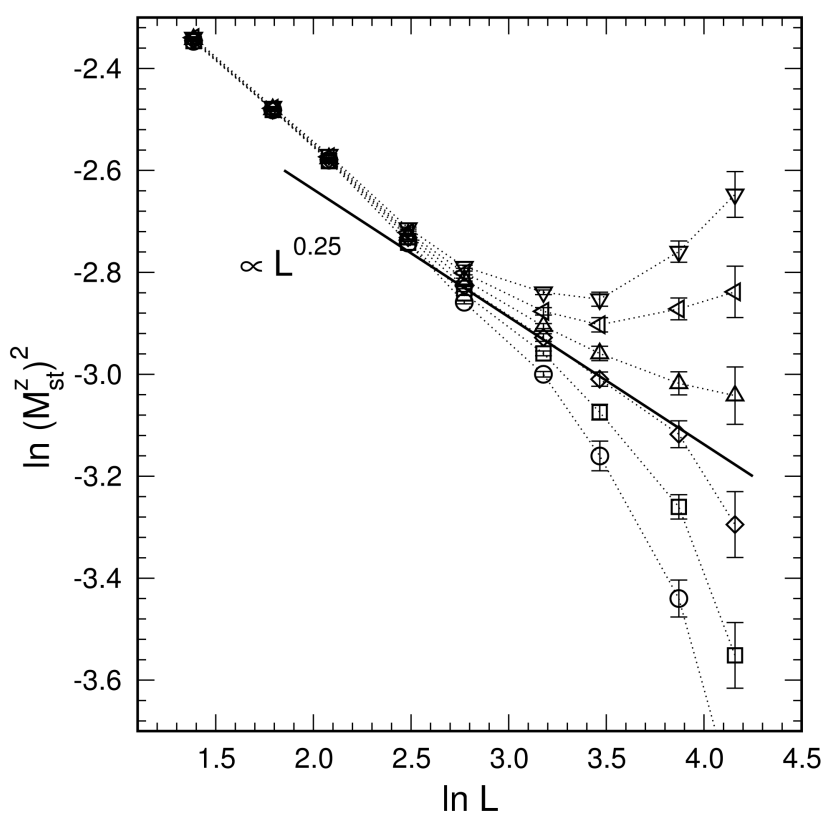

FIG. 6: Doubly logarithmic plot of the staggered magnetization $\left(M_{s t}^{z}\right)^{2}$ vs. the system size $L$ at $H / J=1.225$ for the temperatures $k_{B} T / J=0.095,0.0955,0.096,0.0965,0.097$, and 0.0975 (from bottom to top). The straight line proportional to $L^{\frac{1}{4}}$ illustrates the expected finite-size behavior close to a continuous transition of Ising type.

two peaks, is provided. As depicted in Fig. 5 the positions of the maxima as a function of the inverse system size exhibit a curvature, which becomes more pronounced for larger lattices. In contrast, in the previous analysis $\frac{18}{}$ at the same temperature, linear dependences of the peak positions as a function of $1 / L$ had been presumed, leading to distinct two peaks in the thermodynamic limit. We conclude, that the previous claims of a first-order transition at $k_{B} T / J=0.13$ and of the existence of a tricritical point at $k_{B} T_{\mathrm{t}} / J \approx 0.141$ needs to be viewed with care. Indeed, the tricritical point seems, if it exists at all, to be shifted towards lower temperatures.

In the previous work ${ }^{18}$ a direct transition of first order between the $\mathrm{AF}$ and $\mathrm{SF}$ phases has been suggested to take place at lower temperatures, $k_{B} T / J \leq k_{B} T_{\mathrm{ce}} / J \approx 0.118$. To check this suggestion we studied the system at constant field $H / J=1.225$, where such a direct transition would occur, see Fig. 4. Calculating the expectation values of the different magnetizations as well as the corresponding histograms we obtain an estimate of the critical temperature of the $\mathrm{AF}$ phase, $k_{B} T_{\mathrm{AF}}=0.09625 \pm 0.0005$.

Surprisingly, approaching the transition from the AF phase, the finite-size behavior of the squared staggered magnetization $\left(M_{\mathrm{st}}^{z}\right)^{2}$, being the $\mathrm{AF}$ order parameter, is still consistent with a continuous transition in the Ising universality class: As illustrated in Fig. 6 the asymptotic region is very narrow, similar to the observations in the 
classical model $\stackrel{15,16}{[}$ The dependence on the system size seems to obey $\left(M_{\mathrm{st}}^{z}\right)^{2} \propto L^{1 / 4}$ right at the transition, as expected for the Ising universality class. ${ }^{27}$

Furthermore, approaching the transition from the SF phase, an analysis of the spin-stiffness $\rho_{s}$ at the same field value of $H / J=1.225$ results in about the same transition temperature, $k_{B} T_{\mathrm{SF}} / J=0.09625 \pm 0.001$. Thence, there may be either a unique transition between the $\mathrm{SF}$ and $\mathrm{AF}$ phases, or, as observed in the classical case, an extremely narrow intervening phase, with phase boundaries of Ising and Kosterlitz-Thouless (KT) type.

To determine, whether a KT transition describes the disordering of the SF phase, we check the theoretical prediction 28,29 that for the infinite system the spinstiffness is finite within the SF phase, takes on a universal value at the $\mathrm{KT}$ transition related to $T_{\mathrm{KT}}$ by

$$
\rho_{s}\left(T=T_{\mathrm{KT}}, L=\infty\right)=\frac{2}{\pi} k_{B} T_{\mathrm{KT}},
$$

and discontinuously vanishes in the disordered phase. As depicted in Fig. 7 the spin-stiffness $\rho_{s}$ at $T=T_{\mathrm{SF}}$ seems to be, at first sight, significantly larger than the KTcritical value given by Eq. (91). Indeed, in the earlier study $\underline{18}$ it has been argued, based on similar observations, that there is a direct first order AF to SF transition. However, the finite-size effects close to the transition deserve a careful analysis: For the KT scenario, renormalization group calculations ${ }^{30,31}$ predict the asymptotic size dependence at $T=T_{\mathrm{KT}}$ to obey

$$
\begin{aligned}
& \rho_{s}\left(T=T_{\mathrm{KT}}, L\right)= \\
& \rho_{s}\left(T=T_{\mathrm{KT}}, L=\infty\right)\left(1+\frac{1}{2 \ln L-C_{0}}\right),
\end{aligned}
$$

where $C_{0}$ denotes an apriorily unknown, non-universal, parameter. By studying the quantity ${ }^{32}$

$$
C(L)=-2\left[\left(\frac{\pi \rho_{s}}{k_{B} T}-2\right)^{-1}-\ln L\right],
$$

which, according to Eqs. 9 and 10, converges for $L \rightarrow \infty$ and $T=T_{\mathrm{KT}}$ to the value $C_{0}$ at a $\mathrm{KT}$ transition, we obtain a rough estimate of $C_{0} \approx 5$. A prediction of the finite-size behavior at $T_{\mathrm{SF}}$ is obtained by inserting this value, $C_{0}=5$, into Eq. (10). Comparing the data of the spin-stiffness $\rho_{s}$ in the direct vicinity of the boundary of the SF phase with the prediction according to the KT theory, see Fig. 7 b), one may conclude that the lattice sizes accessible by simulations, $L \leq 64$, seem to be too small to capture the asymptotic finite-size behavior. In any event, in case of a KT transition, the spin-stiffness $\rho_{s}$ drops asymptotically very rapidly to its universal critical value as a function of system size, being consistent with the relatively large values for the simulated finite lattices. Thus, a scenario with a KT transition between the SF and a narrow intervening disordered phase cannot be ruled out by the present large-scale simulations down to temperatures as low as $k_{B} T / J=0.09625 \pm 0.001$.
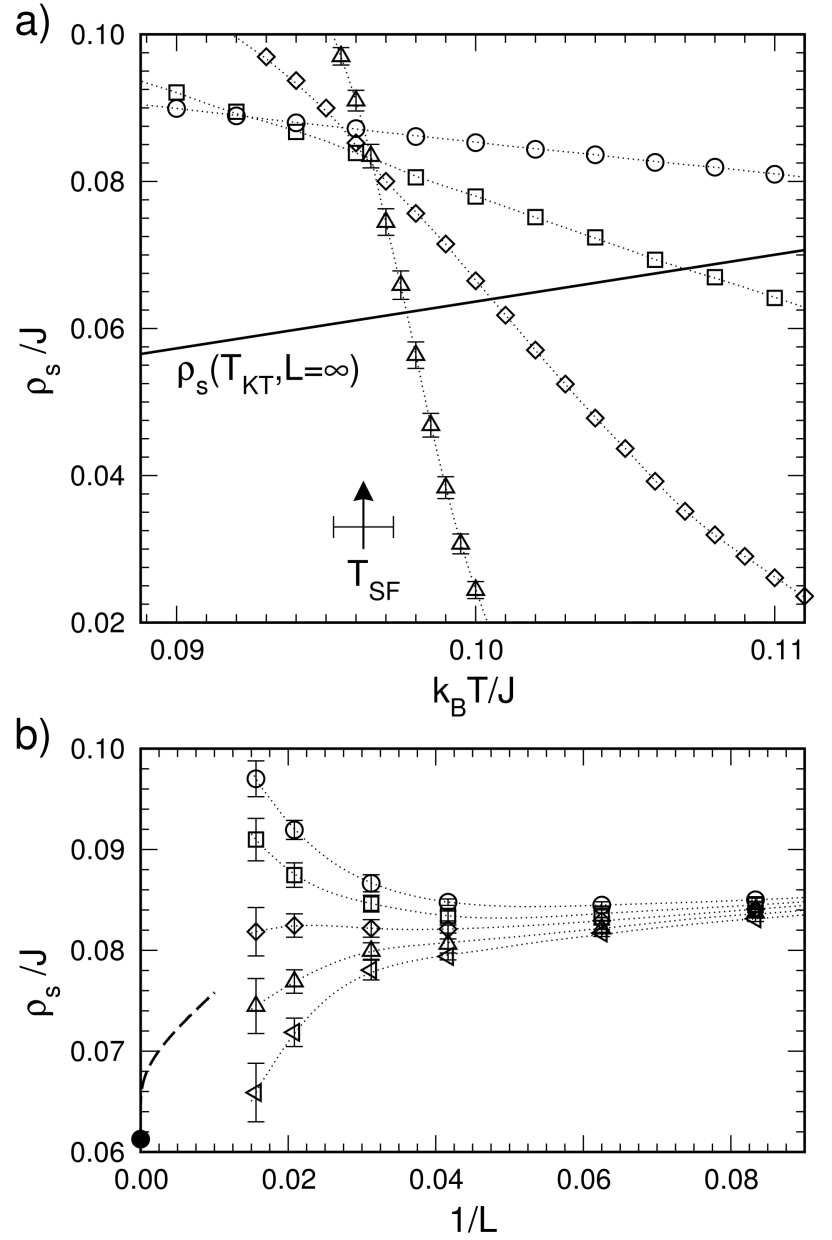

FIG. 7: a) Spin stiffness $\rho_{s} / J$ vs. temperature $k_{B} T / J$ for the different system sizes $L=8$ (circles), 16 (squares), 32 (diamonds), and 64 (triangles). The straight line denotes the critical value of the spin-stiffness according to the formula of Nelson and Kosterlitz ${ }^{29}$, see Eq. (9).

b) Finite-size behavior of the spin-stiffness $\rho_{s} / J$ at $H / J=$ 1.225 as a function of the inverse system size, $1 / L$ for the temperatures $k_{B} T / J=0.0955,0.096,0.0965,0.097$, and 0.0975 (from top to bottom). The dashed curve illustrates the estimated asymptotic behavior according to Eq. (10) with $k_{B} T_{\mathrm{KT}} / J=0.09625$ and $C_{0}=5$, the corresponding critical value $\rho_{s}\left(T_{\mathrm{KT}}, L=\infty\right) \approx 0.0613$ is marked by the filled circle.

Of course, it is desirable to quantify the role of biconical fluctuations in the quantum case as well. However, accessing the probability distributions of the tilt angles studied in Sect. II for the quantum case is beyond the scope of the present numerical analysis.

\section{SUMMARY}

We studied the classical and quantum, $S=\frac{1}{2}$, versions of the XXZ Heisenberg antiferromagnet on the square lattice in an external field along the easy axis. The model 
is known to display ordered $\mathrm{AF}$ and $\mathrm{SF}$ as well as disordered, paramagnetic phases. Here we focused attention to the region of the phase diagram near and below the temperature where the two boundary lines between the AF and the SF phases and the disordered phase approach each other, meeting eventually at a triple point. We performed Monte Carlo simulations, augmented, in the classical case, by a ground state analysis.

In the classical version, we presented first direct evidence for the importance of biconical structures in the XXZ model. Indeed, such configurations do exist already as ground states at the critical field $H_{\mathrm{c} 1}$, separating the $\mathrm{AF}$ and SF phases. The interdependence of the two tilt angles, characterizing the biconical ground states, persists at finite temperatures, in the region where the narrow phase between the AF and SF phases is expected to occur. Indeed, the joint probability distribution of the tilt angles at neighboring sites demonstrates the thermal significance of those configurations. Previous arguments on $O(3)$ symmetry in that region and down to zero temperature thus have to be viewed with care. The results of the present simulations suggest that, if the biconical configurations do not lead to a stable biconical phase in two dimensions, the narrow intervening phase is a disordered phase characterized by biconical fluctuations. In this sense the "hidden bicritical point" at $T=0$ may then be coined into a "hidden tetracritical point."

In the quantum version, previous simulations sug- gested, on lowering the temperature, the existence of a tricritical point on the boundary line between the $\mathrm{AF}$ and disordered phases, followed by a critical endpoint being the triple point of the $\mathrm{AF}, \mathrm{SF}$ and disordered phases, and eventually by a first-order transition between the $\mathrm{AF}$ and SF phases at sufficiently low temperatures. The present simulations, considering larger system sizes and improved statistics, provide evidence that this scenario, if it exists at all, has to be shifted to lower temperatures than proposed before. Of course, simulations on even larger lattices and lower temperatures would be desirable, but are extremely time consuming.

A clue on possible distinct phase diagrams for the classical and quantum versions may be obtained from an analysis of biconical fluctuations in the quantum case. Experimental studies on signatures of those fluctuations are also encouraged.

\section{Acknowledgments}

Financial support by the Deutsche Forschungsgemeinschaft under grant No. SE 324/4 is gratefully acknowledged. We thank A. Honecker, B. Kastening, R. Leidl, A. Pelissetto, M. Troyer, and E. Vicari for useful discussions and information.
1 M. E. Fisher and D. R. Nelson, Phys. Rev. Lett. 32, 1350 (1974); D. R. Nelson, J. M. Kosterlitz, and M. E. Fisher, Phys. Rev. Lett. 33, 813 (1974); J. M. Kosterlitz, D. R. Nelson, and M. E. Fisher, Phys. Rev. B 13, 412 (1976).

${ }^{2}$ H. Rohrer, Phys. Rev. Lett. 34, 1638 (1975).

3 K. Binder and D. P. Landau, Phys. Rev. B 13, 1140 (1976); D. P. Landau and K. Binder, Phys. Rev. B 24, 1391 (1981).

${ }^{4}$ K. Takeda and K. Koyama, J. Phys. Soc. Jpn. 52, 648 (1983); J. Phys. Soc. Jpn. 52, 656 (1983).

${ }^{5}$ H. J. M. de Groot and L. J. de Jongh, Physica B 141, 1 (1986).

${ }^{6}$ R. A. Cowley, A. Aharony, R. J. Birgeneau, R. A. Pelcovits, G. Shirane, and T. R. Thurston, Z. Phys. B 93, 5 (1993).

7 R. van de Kamp, M. Steiner, and H. Tietze--Jaensch, Physica B 241-243, 570 (1997).

8 R. J. Christianson, R. L. Leheny, R. J. Birgeneau, and R. W. Erwin, Phys. Rev. B 63, 140401(R) (2001).

${ }^{9}$ K. Ohgushi and Y. Ueda, Phys. Rev. Lett. 95, 217202 (2005).

10 A. Cuccoli, G. Gori, R. Vaia, and P. Verrucchi, J. Appl. Phys. 99, 08H503 (2006).

11 P. Calabrese, A. Pelissetto, and E. Vicari, Phys. Rev. B 67, 054505 (2003).

12 C. J. Gorter and T. Van Peski-Tinbergen, Physica 22, 273 (1956).

13 H. Matsuda and T. Tsuneto, Prog. Theor. Phys., Supp. 46, 411 (1970).

14 K.-S. Liu and M. E. Fisher, J. Low. Temp. Phys. 10, 655
(1973).

15 M. Holtschneider, W. Selke, and R. Leidl, Phys. Rev. B 72, 064443 (2005).

16 C. Zhou, D. P. Landau, and T. C. Schulthess, Phys. Rev. B 74, 064407 (2006).

17 A. Pelissetto and E. Vicari, cond-mat/0702273 (2007).

18 G. Schmid, S. Todo, M. Troyer, and A. Dorneich, Phys. Rev. Lett. 88, 167208 (2002).

19 M. Kohno and M. Takahashi, Phys. Rev. B 56, 3212 (1997).

20 S. Yunoki, Phys. Rev. B 65, 092402 (2002).

21 A. D. Bruce and A. Aharony, Phys. Rev. B 11, 478 (1975); D. Mukamel, Phys. Rev. B 14, 1303 (1976); E. Domany and M. E. Fisher, Phys. Rev. B 15, 3510 (1977).

22 R. Leidl and W. Selke, Phys. Rev. B 70, 174425 (2004); R. Leidl, R. Klingeler, B. Büchner, M. Holtschneider, and W. Selke, Phys. Rev. B 73, 224415 (2006).

23 A. W. Sandvik and J. Kurkijärvi, Phys. Rev. B 43, 5950 (1991); A. W. Sandvik, J. Phys. A 25, 3667 (1992).

24 O. F. Syljuåsen and A. W. Sandvik, Phys. Rev. E 66, 046701 (2002).

${ }^{25}$ K. Hukushima and K. Nemoto, J. Phys. Soc. Jpn. 65, 1604 (1996).

26 P. Sengupta, A. W. Sandvik, and D. K. Campbell, Phys. Rev. B 65, 155113 (2002).

27 L. Onsager, Phys. Rev. 65, 117 (1944).

28 J. M. Kosterlitz and D. J. Thouless, J. Phys. C 6, 1181 (1973).

29 D. R. Nelson and J. M. Kosterlitz, Phys. Rev. Lett. 39, 
1201 (1977).

30 P. Minnhagen and H. Weber, Physica B 152, 50 (1988).

31 H. Weber and P. Minnhagen, Phys. Rev. B 37, 5986 (1988).
${ }^{32}$ K. Harada and N. Kawashima, Phys. Rev. B 55, R11949 (1997). 